\title{
EL CRECIMIENTO DE LA INFORMACIÓN Y LA TOMA DE DECISIONES
}

\section{GROWTH OF INFORMATION AND DECISION-MAKING}

Marlena León Mendoza, PhD. Doctora en Ciencias de la Educación por la Universidad de La Habana (Cuba). Máster en Administración de Empresas. Master of Management. Vicerrectora Académica y docente titular de la Universidad de Especialidades Espíritu Santo (UEES). Docente titular de la Universidad Tecnológica ECOTEC, Ecuador. mleon@uees.edu.ec

\section{ARTÍCULO DE REFLEXIÓN}

Recibido: 8 de octubre de 2016

Aceptado: 10 de febrero de 2017

\section{RESUMEN}

En el artículo se expone un estudio comparado de la necesidad de utilizar la información en la toma de decisiones. Se presenta un análisis sobre los sistemas de información gerencial y su relación con la mejora de la productividad y la comunicación interna de las organizaciones públicas y privadas. La investigación concluye con una aplicación práctica implementada en el Municipio de Babahoyo en Ecuador.

Palabras Clave: gobierno electrónico, toma de decisiones, información, sociedad.

\section{ABSTRACT}

The article presents a comparative study of the need to use information in decision making. An analysis is presented on management information systems and their relationship with the improvement of productivity and internal communication of public and private organizations. The research concludes with a practical application implemented in the Municipality of Babahoyo in Ecuador.

Keywords: Electronic government, decision making, information, society. 


\section{INTRODUCCIÓN}

Los estudios sociales de la ciencia y la tecnología, o estudios sobre ciencia, tecnología y sociedad (CTS), constituyen un campo de trabajo en los ámbitos de la investigación académica, la educación y la política pública. CTS se origina hace más de tres décadas a partir de nuevas corrientes de investigación en filosofía y sociología de la ciencia, y de un incremento en la sensibilidad social e institucional sobre la necesidad de una regulación democrática del cambio científico-tecnológico (Nuñez, Alonso, \& Ramirez, 2015).

La finalidad de esos estudios es la de que los ciudadanos puedan desempeñarse mejor en una sociedad altamente tecnificada, a la vez que entiendan sus limitaciones y sus peligros. Esto incluye la capacitación para poder participar en la toma de decisiones en lo que respecta a los temas tecnológicos polémicos con un conocimiento de causa suficiente, lo que no siempre es fácil, dada la complejidad de los factores que inciden sobre tales decisiones.

El crecimiento acelerado de la información debe ser tenido en cuenta en la toma de decisiones para lograr que estas se hagan efectivas. Mediante un estudio comparado, basado en el análisis documental, que incluye una revisión de la literatura especializada, se realiza un estudio de cómo poder asumir la toma de decisiones en el entorno actual.

\section{REVISIÓN TEÓRICA}

\section{Entorno socioeconómico actual}

Las condiciones socioeconómicas actuales imponen un ritmo de aceleración en las transacciones comerciales y productivas, en las que el perfeccionamiento tecnológico de sus aplicaciones, de manera integradora y facilitadora de datos que permitan la toma de decisiones con precisión y claridad en un tiempo relativamente corto con respecto a décadas anteriores, es vital (Cohen, 2013).

El desarrollo del software en los tiempos actuales, ha provocado que casi todas las entidades económicas, productivas y gubernamentales deseen adoptar aplicaciones 
tecnológicas que les ofrezcan la posibilidad de automatizar sus procesos de control de recursos humanos, proyectos y acciones relacionadas con la actividad a la que se dedican y que les permitan cumplir los objetivos que se han trazado para un periodo determinado de tiempo. Apoyando de esta manera el trabajo que realizan los directivos de las instituciones. (Garis, 2010).

De acuerdo a Caiceo (2015), entre las principales tareas de los directivos de una institución está la de tomar decisiones, pero la calidad de las decisiones que se tomen depende directamente de la calidad de la información sobre las que se soportan. Por ello se debe considerar que la toma de decisiones no es un proceso sencillo y requiere de un profundo conocimiento del problema y de las condiciones que lo rodean, de las alternativas disponibles y de las estrategias competitivas que se puedan aplicar, así como de acceso y manejo de información de calidad.

Actualmente, se han desarrollado modelos de información oportuna, los cuales están directamente relacionados con el tipo de sistema de información que posea la compañía donde se implementen, el tiempo de validez que tendrá la información y la confiabilidad de la misma, dependen de las fuentes de donde provienen cada uno de los resultados del sistema de información gerencial. Cabe indicar que por el momento, solamente el $25 \%$ de la información que se obtiene, es utilizada para la toma de decisiones por los ejecutivos, debido a la optimización y al ordenamiento clasificado de la información en función de los planes estratégicos de las empresas y porque en la mayoría de los casos no utilizan sistemas de información gerencial que se complementen con la planificación estratégica de las mismas. Por ello se considera que cada día se hace más necesario que los ejecutivos que utilicen los sistemas de información gerencial para la toma de decisiones, implementen además actividades de seguimiento y control, así como la comprobación de la documentación existente (Dedrick, Gurbaxani, \& Kraemer, 2003).

\section{Los sistemas de información}

Un tipo de sistema de información que ayuda a la toma de decisiones en instituciones de gobierno y que facilita la comunicación con los ciudadanos, son los sistemas de gobierno electrónico (Grönlund, 2005; Moon \& Norris, 2005). De acuerdo a Koh y Prybutok (2003), el Gobierno Electrónico consiste en la utilización de las tecnologías 
de la información y la comunicación (TIC) al servicio del sector público, con el objetivo de aumentar la eficiencia, la transparencia y la participación de los ciudadanos, así también lo plantean Meijer (2007) y Lee et al. (2007).

A partir de esta definición se observa como las operaciones de Gobierno Electrónico sitúan a las TICs como elemento de apoyo que promueve el desarrollo de un mejor gobierno (Del Piazzo \& Viega, 2004). Esto implica que se pueden conseguir mejores niveles de eficacia y eficiencia en el quehacer gubernamental, a través del mejoramiento de los procesos y procedimientos del gobierno, incrementando la calidad de los servicios que se ofrecen a la población, incorporando más y mejor información en los procesos de toma de decisiones y facilitando la coordinación entre las diferentes instancias de gobierno (Carou, Gallardo, \& Verde, 2002; Brusca \& Montesinos, 2006).

\section{Situación del Ecuador}

A esta realidad no se encuentran ajenas las instituciones de gobiernos seccionales del Ecuador, sin embargo, en un diagnóstico preliminar realizado se detectó que en el Municipio de Babahoyo se presenta lo siguiente:

- No existen herramientas tecnológicas que se utilicen para el control de la ejecución de proyectos en los diferentes departamentos. El Excel o el Word son herramientas tecnológicas que no han sido creadas para realizar este tipo de actividades y por ende el nivel de satisfacción con su uso para esta gestión no es el más adecuado.

- En la institución no existe una cultura del uso de indicadores de gestión para controlar el desarrollo de los proyectos y actividades relacionadas. De hecho existe desconocimiento en el más amplio sentido de la palabra en cuanto al significado y la importancia del uso de indicadores de gestión, de donde se desprende que es imprescindible que se capacite al personal en este aspecto.

- El uso de herramientas tecnológicas que faciliten la generación de reportes estadísticos amigables y que permitan a los directivos de la institución conocer visualmente el estado de avance de sus proyectos es limitado, mayormente realizan reportes manuales o con Excel, pero el tiempo que emplean en la elaboración de los mismos es excesivamente alto. 
- La generación de encuestas para medir el nivel de satisfacción de la población en relación a la ejecución de proyectos por parte de la municipalidad es manual, por ende no se realizan casi nunca. De lo cual se deduce que es necesario desarrollar una herramienta que además de llevar el control de la ejecución y comunicarlo en la población, permita encuestar el nivel de satisfacción de manera ágil y totalmente confidencial.

- El personal entrevistado preferiría tener una herramienta de control de medios donde puedan conocer las noticias relacionadas con la Municipalidad de Babahoyo y sobre los diferentes ámbitos de interés para la institución.

- Es necesario contar con una herramienta tecnológica que lleve el control de la ejecución de las tareas que desarrollan los miembros de la municipalidad de Babahoyo.

- Se encuentra una alta disposición de los trabajadores del municipio para utilizar una herramienta tecnológica integradora que les permita llevar el control de los proyectos mediante indicadores de gestión, de las tareas encomendadas, de los diferentes medios de información y de las agendas del personal en ambiente web por las facilidades de uso que ello representa para los integrantes de la institución.

\section{MATERIALES Y MÉTODOS}

\section{Buenas prácticas de gobierno electrónico}

Para atender las necesidades no satisfechas del Municipio de Babahoyo, se hizo un análisis de las aplicaciones de gobierno electrónico que han sido pioneras en diferentes regiones del mundo y del Ecuador, entre las que podemos destacar las siguientes:

- Canadá: Es uno de los países que se encuentra a la cabeza de la innovación del sector público electrónico, pues incursionó en esta área desde finales del año 1980. Para el año 1999, el gobierno de Canadá se fijó el objetivo de ser reconocido en el mundo como "El gobierno más conectado con sus ciudadanos, con canadienses capaces de acceder a toda la información gubernamental y servicios en línea en cualquier lugar o tiempo que desearan..." y lo logró a través de la implementación de campañas de comunicación muy fuertes sobre 
el acceso a las plataformas tecnológicas que tenían al alcance. Casi el $90 \%$ de sus ciudadanos están conectados, esto hace que la transparencia de información se vuelva más fácil y ágil en Canadá (West \& Deitch, 2004).

- Singapur: Se encuentra dentro de la lista de los pioneros mundial en Gobierno Electrónico. Es el primer país en implementar una infraestructura de BroadBand (Banda Ancha) a nivel nacional, gracias a la cual $99 \%$ de los ciudadanos tienen acceso al Internet. En 1981 se inició el primer plan nacional de tecnología de la información para promover el uso de tecnología de información en el Gobierno. Sus planes cada vez han sido más ambiciosos, puesto que desean posicionar al país como uno de los líderes en el uso de las tecnologías de información a nivel global. En 1997, el gobierno creó eCitizen, un portal que es la única vía de entrada de Singapur a la información y servicio del gobierno. Dentro de la información está integrada la educación, la vivienda, trabajos, etc. Tiene en promedio 9.000 .000 de consultas al mes. Actualmente, Singapur ofrece más de 1.600 servicios en línea, los ciudadanos solo tienen que ingresar al portal de eCitizen para acceder a sus servicios (Chadwick, 2003).

- Uruguay: Fue uno de los pioneros en estos sistemas en América Latina mediante el sitio web www.comprasestatales.gub.uy, a través del cual el gobierno mostraba toda la información pertinente a las compras que el estado realiza. Esta fue una gran ventaja para el gobierno debido a la facilidad con la que el estado podía obtener reportes, y tener encapsulada toda la información en un solo sitio. Pero esto no solo fue ventajoso para el gobierno, sino que también presentó una gran oportunidad para los proveedores de poder participar en negocios con el estado, que para muchas empresas era incluso imposible de realizar (Criado \& Ramilo, 2001).

\section{ANÁLISIS DE RESULTADOS Y DISCUSIÓN}

\section{Sistemas de información de Ecuador}

En la Tabla 1, se caracterizan los sistemas de información que utilizan algunas instituciones gubernamentales del Ecuador, entre ellas: Gobierno Nacional del Ecuador, Municipalidad de Quito, Municipalidad de Guayaquil y la Asociación de Municipalidades del Ecuador (AME). Cabe indicar que los criterios que se han 
seleccionado son los siguientes: Objetivos, acciones que puede desarrollar el usuario, ambiente en que se desarrolla, sentido de la comunicación, costo.

Del análisis realizado en Ecuador y otros países se observa que:

- Las instituciones gubernamentales no quedan excluidas de la implementación de sistemas de información gerencial para llevar el control de sus actividades. En este sentido juegan un papel importante los sistemas de gobierno electrónico a través de los cuales se establece una relación BIDIRECCIONAL entre el gobierno y la ciudadanía.

- Los gobiernos más desarrollados en la implementación del gobierno electrónico a nivel mundial son los de Canadá, Nueva Zelanda y Singapur, pues fueron los pioneros en este tipo de implementaciones.

- El gobierno del Ecuador también ha comenzado a incursionar en el gobierno electrónico a través de herramientas tecnológicas como el SIGOB y el SIGEF, actualmente se encuentra en la fase de transacción de manera incipiente, habiendo superado las fases de presencia y de interacción.

- Los gobiernos locales del Ecuador también están comenzando a utilizar herramientas tecnológicas para comunicarse con su población. La forma más comúnmente encontrada es a través de sus páginas webs, en las que ya se pueden realizar consultas de tasas de diferentes rubros municipales y se han establecido formas de comunicación BIDIRECCIONAL.

- La mayoría de las herramientas que se utilizan para comunicarse con la población en general, se ejecutan en ambiente web por las facilidades de uso que representan para el público en general.

- De los gobiernos locales encontrados, el de Quito es el único que cuenta con una plataforma tecnológica que le permite comunicar el estado de avance de su planificación estratégica y el cumplimiento de sus metas a través de la medición de sus indicadores de gestión, pero aún no ha establecido formas de comunicación BIDIRECCIONAL con sus pobladores.

- La AME apoya la implementación de herramientas tecnológicas en sus municipios a través de su página web, en la que se pueden descargar diferentes herramientas, así como sus actualizaciones, pero aún no pone a disposición de sus usuarios herramientas de medición y control del 
cumplimiento y desarrollo de sus proyectos, por lo cual sería interesante firmar un acuerdo con ella para contribuir al desarrollo del país.

- Existe una necesidad a nivel nacional de sistemas de información gerencial que apoyen la gestión de las instituciones gubernamentales y no gubernamentales, cuyos precios de comercialización no sean excesivos y se desarrollen a la medida de sus necesidades. 
Tabla 1. Caracterización de las plataformas de gobierno electrónico en Ecuador

Institución

\section{Objetivos de}

implementación

\section{Acciones que \\ puede realizar el}

$$
\text { usuario }
$$
Gobierno Naciona
del Ecuador

Visualizar el estado de los proyectos proyectos

realizados

realizar

Ecuador.

por que realiza

el gobierno nacional.

$$
\text { al. }
$$

el nacional

se desarrolla

\section{Sentido de la}

comunicación

as aplicaciones

gobierno

desarrollan

de la

ambiente WEB.

Mejorar la
comunicación entre
el gobierno y la

Enviar sus Según

el gobierno y la

ciudadanía.

opiniones sobre los información

(a)

Brindar servicios de

registro

transaccionales,

control y consultas

por parte de las

instituciones

beneficios

del

desarrollo

del recogida, cada día proyecto.

incrementa el grado de interacción de la

Enviar correos

electrónicos a los

encargados de cada

población con las

plataformas del actualizada

gobierno nacional función de sus

proyecto.

(SIGOB y SIGEF) demandas

pues se ha creado

de los funcionarios. una necesidad de

de transparentar la de opinión.

información

aumentar

y Calificarse

la proveedor

como

$$
\text { recibir }
$$

eficiencia

servicios para el

estado.

Asociación de
Municipalidades
del Ecuador

Servir de

información

consulta

Descargar

de

determinados

automatizada sobre

aplicaciones

instalar

en

diferentes

municipios del país

La aplicación

para desarrolla

ambiente

aunque

descargan
El sentido de la

comunicación de las

aplicaciones

del inversión

es

pues permiten que

ciudadanos

opiniones a través

del portal, sí como

también recibir

información

as que




\begin{tabular}{|c|c|c|c|c|c|}
\hline & $\begin{array}{l}\text { municipal y absolver } \\
\text { consultas que le } \\
\text { fueren hechas por } \\
\text { los gobiernos } \\
\text { locales. }\end{array}$ & $\begin{array}{l}\text { Descargar } \\
\text { actualizaciones de } \\
\text { las aplicaciones a } \\
\text { servicio de los } \\
\text { municipios } \\
\text { Realizar búsquedas } \\
\text { de instituciones } \\
\text { para obtener ayuda } \\
\text { y cooperación } \\
\text { internacional en el } \\
\text { desarrollo de } \\
\text { proyectos } \\
\text { Acceder a } \\
\text { información vigente } \\
\text { de leyes y } \\
\text { reglamentaciones } \\
\text { que aplican a la } \\
\text { gestión municipal }\end{array}$ & $\begin{array}{l}\text { realizan los usuarios } \\
\text { son ejecutables } \\
\text { solamente ren } \\
\text { ambiente Windows, } \\
\text { por lo que sus } \\
\text { resultados } \\
\text { visualizan se } \\
\text { solamente a nivel } \\
\text { local. }\end{array}$ & $\begin{array}{l}\text { UNIDIRECCIONAL, } \\
\text { pues solamente } \\
\text { fluye en el sentido } \\
\text { de la AME hacia las } \\
\text { municipalidades. } \\
\text { Cabe indicar que } \\
\text { esto surge de las } \\
\text { asambleas y } \\
\text { reuniones constante } \\
\text { que lleva a cabo la } \\
\text { AME con los } \\
\text { municipios del país, } \\
\text { por lo que aunque } \\
\text { en la aplicación no } \\
\text { se muestre, si existe } \\
\text { un alto grado de } \\
\text { comunicación entre } \\
\text { la AME y los } \\
\text { municipios. }\end{array}$ & $\begin{array}{lr}\text { de los municipios } \\
\text { tienen un } & \text { costo } \\
\text { promedio } & \text { de } \\
100,000.00 & \text { dólares. } \\
\text { Debemos asumir } \\
\text { que el paquete } \\
\text { completo r de } \\
\text { aplicaciones tiene } \\
\text { un valor aproximado } \\
\text { de } \mathbf{3 0 0 , 0 0 0 . 0 0} \\
\text { dólares, los cuales } \\
\text { se dividen entre } \\
\text { todos los municipios } \\
\text { en función de su } \\
\text { tamaño } \\
\text { presupuesto, por } \\
\text { ello resulta una } \\
\text { opción } \\
\text { conveniente, muy } \\
\text { especialmente para } \\
\text { los municipios } \\
\text { pequeños. }\end{array}$ \\
\hline $\begin{array}{l}\text { Municipio de } \\
\text { Quito }\end{array}$ & $\begin{array}{l}\text { Conocer el estado y } \\
\text { evolución del plan } \\
\text { integral de } \\
\text { desarrollo de Quito } \\
\text { - Equinoccio } 21\end{array}$ & $\begin{array}{ll}\text { Visualizar } & \text { los } \\
\text { proyectos } & \\
\text { relacionados } & \text { con } \\
\text { los diferentes } & \text { ejes } \\
\text { de desarrollo. } & \end{array}$ & $\begin{array}{l}\text { La aplicación se } \\
\text { desarrolla en } \\
\text { ambiente WEB para } \\
\text { el usuario final, pero } \\
\text { al interior del } \\
\text { municipio de Quito, } \\
\text { la información se } \\
\text { ingresa en la base } \\
\text { de datos a través de } \\
\text { una aplicación de } \\
\text { Windows en la que }\end{array}$ & $\begin{array}{l}\text { El sentido de la } \\
\text { comunicación es } \\
\text { UNIDIRECCIONAL, } \\
\text { del municipio a la } \\
\text { población, pues los } \\
\text { usuarios no tienen } \\
\text { opciones que les } \\
\text { permitan enviar } \\
\text { mensajes o criterios } \\
\text { a las autoridades } \\
\text { municipales }\end{array}$ & $\begin{array}{l}\text { De la información } \\
\text { que se logró } \\
\text { recopilar, } \\
\text { encontró que el } \\
\text { precio del Balanced } \\
\text { ScoreCard } \\
\text { Participativo para el } \\
\text { Municipio de Quito } \\
\text { fue de } 87,000.00 \\
\text { dólares y tuvo un } \\
\text { tiempo de }\end{array}$ \\
\hline
\end{tabular}




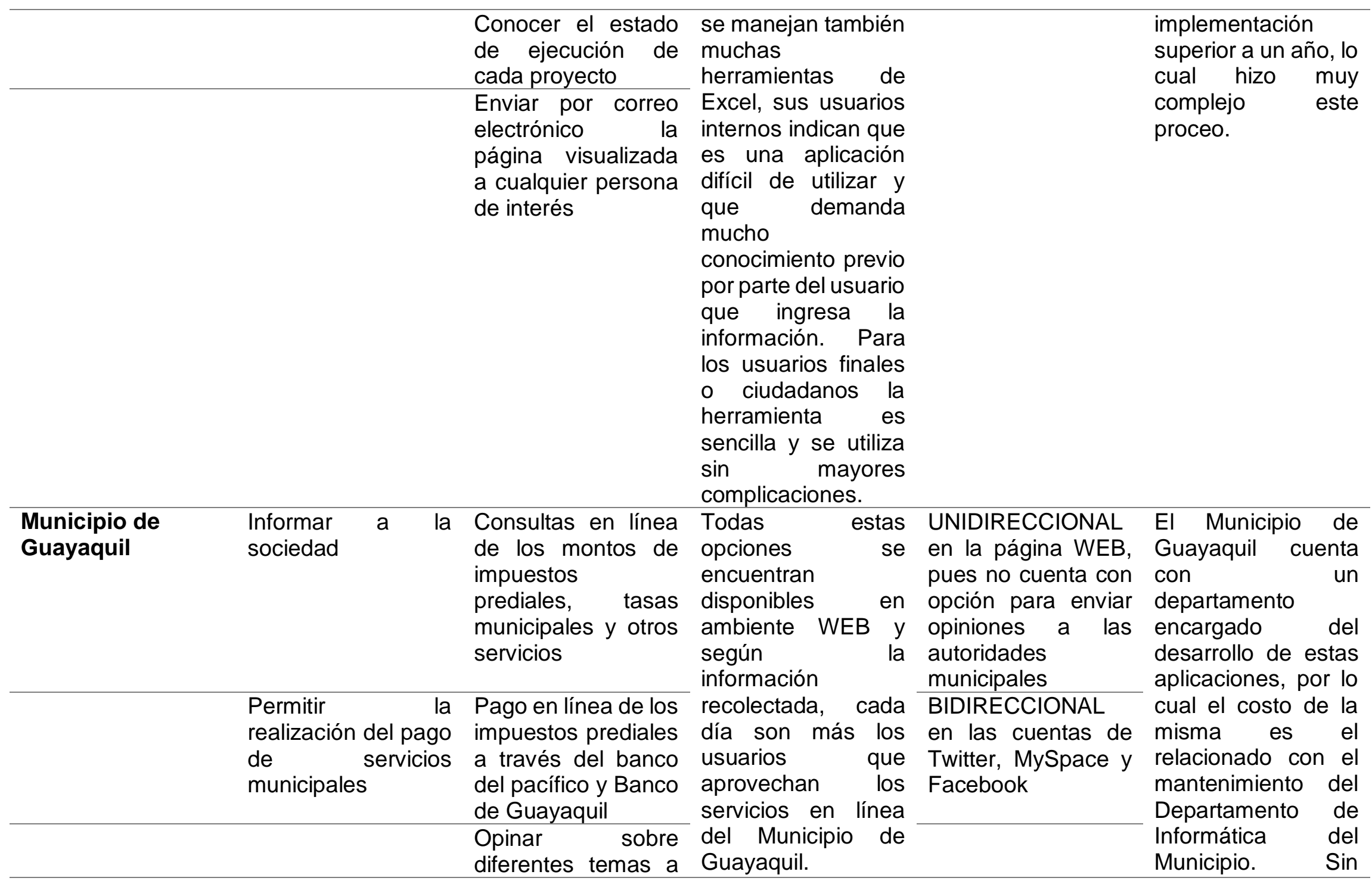


Revista Científica ECOCIENCIA

\begin{tabular}{|c|c|c|}
\hline & $\begin{array}{l}\text { través de cuentas } \\
\text { en Twitter, MySpace } \\
\text { y Facebook }\end{array}$ & $\begin{array}{llr}\text { embargo } & \text { se } & \text { debe } \\
\text { aclarar } & \text { que } & \text { no } \\
\text { cuentan } & & \text { con }\end{array}$ \\
\hline & $\begin{array}{ll}\text { Conocer } & \text { la } \\
\text { información } & \\
\text { relacionada con el } \\
\text { cumplimiento de la } \\
\text { Ley } \\
\text { Transparencia de }\end{array}$ & $\begin{array}{l}\text { herramientas que } \\
\text { les permitan llevar el } \\
\text { control del avance } \\
\text { de los proyectos en } \\
\text { ejecución de } \\
\text { manera visual. }\end{array}$ \\
\hline
\end{tabular}

Fuente: Elaboración propia 


\section{Propuesta de solución al problema estudiado.}

Luego de identificar las principales características de los sistemas de información gerencial que se están aplicando en las diferentes instituciones gubernamentales del país, de Latinoamérica y del mundo, se diseñó un software para que sea implementado inicialmente en el Municipio de Babahoyo y que podrá ser utilizado posteriormente en otros municipios del país y en empresas del sector público y privado. Este es un programa que moderniza las actividades administrativas y de control con procesos de gobierno electrónico, está dirigido a instituciones que quieran operar en forma eficiente y efectiva en el manejo de sus objetivos y responsabilidades.

Los módulos que componen la plataforma desarrollada se detallan a continuación:

\section{Gestión de Proyectos y Actividades Relacionadas (GEPAR)}

Este módulo tiene como objetivo principal garantizar el cumplimiento de los programas y metas trazados en la institución, permitiendo además un mejor control de los entes que intervienen en el desarrollo de los mismos. GEPAR contribuirá a un desempeño más abierto y transparente de los proyectos que se están realizando, permitiendo una operación ágil, sistematizada, para funcionarios de la institución y para que la ciudadanía en general pueda tener acceso y conocimiento de la gestión realizada por la Institución. Además, permitirá que las principales autoridades de la institución cuenten con información al día sobre el estado de programas y actividades planificados, facilitando procesos de trabajo eficientes orientados hacia objetivos y metas mediante la utilización de las tecnologías de la información.

\section{Administración y Control de Tareas (ACT)}

Este módulo permitirá realizar un seguimiento detallado de las tareas a realizarse en cada departamento de la institución, identificando nodos (departamentos, direcciones o empleados) improductivos, lo cual generará un ahorro de tiempo y dinero y el mejoramiento de los procesos en la institución mediante la definición de las mejores rutas para cumplir 
un objetivo. Lograr mejorar la comunicación interna, tener un mejor seguimiento de las actividades que se desarrollan en la institución, ahorrar recursos, mejorar la organización, documentar los procesos en cada dependencia y medir los índices de productividad, son los beneficios que se obtendrán con la implementación de este módulo en cualquier institución.

\section{Seguimiento de Medios (SM)}

Permite a la autoridad y a la institución la actualización en la realidad nacional e internacional en ámbitos de interés político, social y económico. Este módulo generará reportes resumidos con las noticias y hechos que han ocurrido y que son de mayor interés para la comunidad en general. EI SM articula enfoques conceptuales, métodos de trabajo e instrumentos tecnológicos avanzados para asegurar, a partir de un proceso de monitoreo de los medios de comunicación (web, periódicos, radio y televisión), el registro y la sistematización de información de interés para la institución en la que se implemente. Este módulo además, tiene la utilidad de difundir a la ciudadanía los resultados de la gestión institucional como una página promocional de la institución. Facilita el trabajo de Relaciones Públicas y difunde periódicamente la información de los integrantes del sistema.

\section{Control de Agendas (CM)}

Con este módulo se permite a la organización llevar una planificación de las actividades de cada uno de sus integrantes de la Institución principalmente del Presidente, asesores, y de sus directores departamentales. De esta manera, en cualquier momento el líder de la institución puede tener conocimiento sobre las acciones de sus funcionarios y del apoyo entregado al cumplimiento de los objetivos institucionales. La información se controla en base a las necesidades y definiciones de la autoridad. 


\section{CONCLUSIONES}

- El estudio realizado permitió caracterizar el entorno actual en cuanto al crecimiento de la información y se argumenta la necesidad de su uso en la toma de decisiones para lograr mayor calidad de las mismas.

- En un mundo globalizado, el estudio de buenas prácticas adquiere una mayor relevancia para generalizar resultados en diferentes contextos haciendo las correcciones oportunas. Las experiencias en el gobierno electrónico son un ejemplo ilustrativo de esta afirmación.

- El Ecuador dispone de un sistema de información apropiado para aplicar el desarrollo científico en la gestión administrativa para mejorar la toma de decisiones.

- La plataforma propuesta, integrada por cuatro módulos fuertemente interconectados, es una adecuada respuesta a los planteamientos analizados en este estudio, donde se integra el desarrollo científico, las necesidades de la sociedad y el papel del gobierno ecuatoriano.

\section{REFERENCIAS BIBLIOGRÁFICAS}

Brusca, I., \& Montesinos, V. (2006). Are citizens significant users of government financial information. Public Money \& Management , 26, 205.

Caiceo, J. (2015). Gerencia. Obtenido de EMB: http://www.emb.cl/gerencia/articulo.mvc?xid=372.

Carou, H., Gallardo, J., \& Verde, J. (2002). Democracia Digital: Límites y oportunidades. En Capítulo 4. Madrid: Trotta.

Chadwick, A. (2003). Bringing E-Democracy back in why in mathers for future research on eGovernance. Social Science Computer Review , 21 (4), 443-445.

Cohen, A. (2013). Sistemas de Información Gerencial para los negocios. Mc Graw Hill.

Criado, J., \& Ramilo, M. (2001). E-Administración: ¿un Reto o una Nueva Moda para las Administraciones del Siglo XXI? Algunos Problemas y Perspectivas de Futuro en torno a Internet y las TIC en las Administraciones Públicas. Revista Vasca de Administración Pública , 61 (1), 11-43. 
Dedrick, J., Gurbaxani, V., \& Kraemer, K. (2003). Information technology and economic performance: A critical review of the empirical evidence. ACM Computing Surveys , 35 (1), 1-28.

Del Piazzo, C., \& Viega, M. (2004). Lecciones de Derecho Telemático. Montevideo: Fundación de Cultura Universitaria.

Garis, A. (2010). Lógica temporal en verificación de modelos de software. Origen y evolución hasta los tiempos actuales. Fundamentos en Humanidades, 151-161.

Grönlund, A. (2005). State of the art in e-gov research: Surveying conference publications. International Journal of Electronic Governement Research. , 1 (4), 1-25.

Koh, C., \& Prybutok, V. (2003). The three-ring model and development of an instrument for measuring dimensions of e-government functions. Journal of Computer Information Systems , 33 (3), 34-42.

Lee, J., \& Kim, J. (2007). Grounded theory analysis of e-government initiatives: Exploring perceptions of governement authorities. Governement Information Quaterly , 24 (1), 135-147.

Meijer, A. (2007). Publishing public performance results on the internet: Do stakeholders use the internet to hold dutch public service organizations to accounts? Governement, Information Quarterly , 24 (1), 165-185.

Moon, J., \& Norris, D. (2005). Does managerial orientation matter? The adoption of reinventing government and e-government at the municipal level. Info Systems, 15, 43-60.

Nuñez, J., Alonso, L., \& Ramirez, G. (2015). La filosofía de la ciencia entre nosotros: evolución, institucionalización y circulación de conocimientos en Cuba. Revista iberoamericana de Ciencia, Tecnología y Sociedad , $10(28), 147-158$.

West, D., \& Deitch, A. (2004). Desarrollo de estrategias de gobierno electrónico en Chile, Canadá y Brasil. Foro de mejores prácticas de las Américas. Instituto para la conectividad en las Américas. 